\title{
CircRNA UBAP2 Serves as a Sponge of miR-1294 to Increase Tumorigenesis in Hepatocellular Carcinoma through Regulating c-Myc Expression
}

Min-Cheng Yu

Zhongshan Hospital Fudan University

Guang-Yu Ding

Zhongshan Hospital Fudan University

Pei-Yao Fu

Zhongshan Hospital Fudan University

Peng Ma

Zhongshan Hospital Fudan University

Xiao-Dong Zhu

Zhongshan Hospital Fudan University

Jia-Bin Cai

Zhongshan Hospital Fudan University

Ying-Hao Shen

Zhongshan Hospital Fudan University

Jian Zhou

Zhongshan Hospital Fudan University

Jia Fan

Zhongshan Hospital Fudan University

Hui-Chuan Sun

Zhongshan Hospital Fudan University

Ming Kuang

Zhongshan Hospital Fudan University

Cheng Huang ( $\nabla$ huang.cheng@zs-hospital.sh.cn )

Zhongshan Hospital Fudan University

\section{Research}

Keywords: Circular RNA, Hepatocellular carcinoma, miRNA, c-Myc

Posted Date: August 13th, 2020

DOI: https://doi.org/10.21203/rs.3.rs-49281/v2 
License: (c) (i) This work is licensed under a Creative Commons Attribution 4.0 International License. Read Full License

Version of Record: A version of this preprint was published at Carcinogenesis on January 1st, 2021. See the published version at https://doi.org/10.1093/carcin/bgab068. 
The authors have withdrawn this preprint from Research Square 